VOL. 32 (1985), 73-82.

\title{
ON EXISTENCE THEOREMS FOR DIFFERENTIAL EQUATIONS IN BANACH SPACES
}

\author{
Józef BANÁ́
}

\begin{abstract}
In this paper we show that a number of existence theorems for the Cauchy problem of ordinary differential equations in Banach spaces are only apparent generalizations of the previous ones.
\end{abstract}

\section{Introduction}

In the last years there have appeared a lot of papers containing theorems on the existence of solutions of ordinary differential equations in Banach spaces (see, for example, [7], [10], [11], [13]). In most of those theorems it has been assumed that the right hand side of a differential equation

$$
x^{\prime}=f(t, x), x(0)=x_{0},
$$

is a uniformly continuous function in a Banach space and satisfies a comparison condition of Kamke type translated in terms of a so-called measure of noncompactness. Such conditions have the form

$$
\mu(f(t, X)) \leq \omega(t, \mu(X))
$$

being a natural translation of the comparison condition of the type

$$
\|f(t, x)-f(t, y)\| \leq \omega(t,\|x-y\|) .
$$

Received 14 December 1984. The author would like to thank C. Olech and $G$. Hetzer for helpful conversation and valuable suggestions.

Copyright Clearance Centre, Inc. Serial-fee code: 0004-9727/85 $\$ A 2.00+0.00$. 
The first theorem of such type (involving the function $\omega(t, u)=k u$ ) has been proved by Ambrosetti [1]. Its later generalization has been given by Geobel and Rzymowski [8], Sadovskii [13], Szufla [15], Deimling [6, 7] and so on. It is worth while to note that the mentioned generalizations depend mostly on the assumption of a more general Kamke function $\omega(t, u)$. For instance, Sadovski $i$ has assumed a comparison function of Perron type [13] and Szufla that of Coddington and Levinson type [15]. Conditions guaranteeing the existence of only trivial solutions both of a differential equation $u^{\prime}=\omega(t, u)$ and of an integral inequality $u(t) \leq \int_{0}^{t} \omega(s, u(s)) d s$ have been considered. Also some more general classes of comparison functions have been investigated $[6,7]$.

The main aim of this paper is to show that the generalizations of existence theorems for (1) in the direction of assuming more general comparison functions are only apparent generalizations, because all such theorems are equivalent to that one involving a comparison function of Perron and even Bompiani type (see [16]). This situation is similar to the result of Olech [12] who proved that if the right hand side of the equation (1) is continuous then the comparison conditions of Kamke or Coddington and Levinson type are only apparently more general than that of Perron type.

\section{Notation and definitions}

Let $E$ be a real Banach space with the norm $\|\cdot\|$ and the zero element $\Theta$. In this paper we will use the notation from the paper [2]. For example, the symbol $K(x, r)$ will denote the closed ball centered at $x$ and radius $r$ and by $K(X, r)$ we will denote the ball centered at a set $X$ and with radius $r$; that is

$$
K(X, r)=\bigcup_{x \in X} K(x, r) .
$$

The closure of a set $X$, its diameter and the distance between a point $x$ and $X$ are denoted by $\bar{X}$, diam $X$, dist $(x, X)$, respectively. The symbol Conv $X$ denotes the closed convex hull of $X$. For a bounded set $X$ we denote by $\|X\|$ its norm, $\|X\|=\sup [\|x\|: x \in X]$. For subsets of the space $E$ we define the following algebraic operations

$$
X+Y=[x+y: x \in X, y \in Y],
$$




$$
a X=[a x: x \in X], a \in R .
$$

Further, denote by $M_{E}$ (or shortly $M$ ) the family of all nonempty and bounded subsets of $E$ and by $N$ its subfamily consisting of all relatively compact sets. For $X, Y \in M$ let us put

$$
\begin{aligned}
& d(X, Y)=\inf [r: X \subset K(Y, r)], \\
& D(X, Y)=\max [d(X, Y), d(Y, X)] .
\end{aligned}
$$

The number $D(X, Y)$ is called the Hausdorff distance between $X$ and $Y$. For its properties we refer to [9].

In what follows let $X \in M_{E}$. By a measure of noncompactness of a set $X$ we will understand the number $\mu(X)$ defined in the following way:

$$
\mu(X)=\inf [r>0: X \text { has a finite } r \text {-net in } E] \text {. }
$$

Let us notice that $\mu: M_{E} \rightarrow R_{+}\left(R_{+}=(0,+\infty)\right)$ and is the so-called Hausdorff or ball measure of noncompactness [2]. There exist various definitions of a measure of noncompactness [2], [13], but the above seems to be the most convenient for calculations and is very often used.

Now we quote a few properties of $\mu$ which will be used in the sequel:

$$
\begin{aligned}
& 1^{\circ} \mu(X)=0 \Leftrightarrow X \in N_{E}, \\
& 2^{\circ} \quad X \subset Y \Rightarrow \mu(X) \leq \mu(Y), \\
& 3^{\circ} \mu(a X)=|a| \mu(X), a \in R, \\
& 4^{\circ} \mu(X+Y) \leq \mu(X)+\mu(Y), \\
& 5^{\circ} \mu(\operatorname{Conv} X)=\mu(X), \\
& 6^{\circ} \mu(X \cup Y)=\max [\mu(X), \mu(Y)], \\
& 7^{\circ} \mu(K(x, r))=r, \\
& 8^{\circ}|\mu(X)-\mu(Y)| \leq D(X, Y) .
\end{aligned}
$$

The proofs may be found in [2], [13], for example.

\section{Comparison functions of Kamke type}

In the literature one can meet a lot of conditions guaranteeing the uniqueness of solutions of the Cauchy problem (1). Most of these 
conditions have the "comparison" form [16], that is, one requiring a comparison problem with respect to (1) having a solution with specific properties. In the sequel we will discuss such conditions.

Let us denote by $T$ a fixed positive real number and let $J=(O, T), J_{0}=(0, T)$. Denote by $\Omega$ an arbitrary open subset of a Given Banach space $E$ and fix $x_{0} \in \Omega$. Let $f: J \times \Omega \rightarrow E$ be an arbitrary function.

DEFINITION. A function $\omega: J \times R_{+} \rightarrow R_{+}$(or $\omega: J_{0} \times R_{+} \rightarrow R_{+}$) will be called a Kamke comparison function provided the inequality

$$
\left.\|f(t, x)-f(t, y)\| \leq \omega(t,\|x-y\|), x, y \in \Omega, t \in J \text { (or } t \in J_{0}\right),
$$

together with some additional assumptions concerning the function $\omega$, guarantees that the problem (1) has at most one solution.

Further we quote some frequently used classes of Kamke comparison functions.

Class A. This class contains all functions $\omega(t, u)=\omega: J \times R_{+} \rightarrow R_{+}$continuous and nondecreasing with respect to $u$, having the property $\omega(t, 0) \equiv 0$ and such that $u(t) \equiv 0$ is the only continuous function on $J$ satisfying the inequality

$$
u(t) \leq \int_{0}^{t} \omega(s, u(s)) d s, u(0)=0 .
$$

Class $B$. A continuous function $\omega: J \times R_{+} \rightarrow R_{+}$belongs to the class $B$ provided it is nondecreasing with respect to the second variable, $\omega(t, 0) \equiv 0$ and the only differentiable function on $J$ which satisfies on $J$ the equation $u^{\prime}=\omega(t, u)$ and the condition $u(0)=0$, is $u(t) \equiv 0$.

class $C$. The function $\omega: J_{0} \times R_{+} \rightarrow R_{+}$belongs to the class $C$ if for any $\varepsilon>0$ there exists $\delta>0$ and a sequence $t_{i} \rightarrow 0+, t_{i}>0$ and a sequence of functions $\rho_{i}:\left\langle t_{i}, T\right\rangle \rightarrow R_{+}$which are differentiable on $\left\langle t_{i}, T\right\rangle$ and satisfy the inequalities $\rho_{i}^{\prime}>\omega\left(t, \rho_{i}\right), \rho_{i}(t) \geq \delta t_{i}$, $0<\rho_{i}(t) \leq \varepsilon$ for $t \in\left\langle t_{i}, T\right\rangle$.

Let us notice that class $B$ was first investigated by Bompiani [4] 
and its generalization was given by Perron. A more general class than those given by Bompiani and Perron was given in 1930 by Kamke. Next, Coddington and Levinson have given their class of comparison functions [5] being more general than the previous ones. It is worth while to mention that the most general class of Kamke comparison functions considered in the mathematical literature is the class $C$ [16]. For other details and references concerning Kamke classes we refer to the monograph [16] (see also [3]).

In what follows we give two theorems which will be used in the sequel. THEOREM 1. $A=B$.

We omit the easy proof (see [16]).

In view of the above theorem we can use classes $A$ and $B$ interchangeably .

THEOREM 2. Let us assume that $\omega_{1}: J \times R_{+} \rightarrow R_{+}$is continuous, nondecreasing with respect to the second variable and $\omega_{1}(t, 0) \equiv 0$. Further let there exist a function $\omega \in \mathcal{C}$ such that $\omega_{1}(t, u) \leq \omega(t, u)$, $(t, u) \in J_{0} \times R_{+}$. Then $\omega_{1} \in A$.

Proof. Suppose, on the contrary, that $\omega_{1} \notin A$ which means, in view of Theorem 1, that there exists a solution of the equation $u^{\prime}=\omega_{1}(t, u)$, $u(0)=0$ and such that $u(T)=\varepsilon>0$. Actually this solution must satisfy the condition $u(t)=o(t)$ as $t \rightarrow 0+$. On the other hand there exists $\delta>0$, a sequence $t_{i} \rightarrow 0, t_{i}>0$ and a sequence of functions $\rho_{i}:\left\langle t_{i}, T\right\rangle \rightarrow R_{+}$which are differentiable on $\left\langle t_{i}, T\right\rangle$ and such that $\rho_{i}^{\prime}>\omega\left(t, \rho_{i}\right), \quad 0<\rho_{i}(t) \leq \varepsilon, \rho_{i}\left(t_{i}\right) \geq \delta t_{i}$ for $t \in\left\langle t_{i}, T\right\rangle$. Hence and with respect to the appropriate theorem on differential inequalities (see, for example, [14], Lemma 6.3, p. 18) it follows that $\rho_{i}(t) \leq u(t)$ for $t \in\left\langle t_{i}, T\right\rangle$, so that $\rho_{i}\left(t_{i}\right)=o\left(t_{i}\right)$ as $i$ tends to infinity. This contradicts the assumption about $\rho_{i}(t)$ and completes the proof. 


\section{Relations among some existence theorems}

For a given Banach space $E$ denote by $C=C(J, E) \quad(J=\langle 0, T\rangle)$ the space of all continuous functions mapping the interval $J$ into the space $E$ with the usual maximum norm. Further, let $\mu$ be a measure of noncompactness in the space $E$. For $X \in M_{C}$ we will write $X(t)=[x(t): x \in X]$.

We recall the following lemma [2].

LEMMA 1. $D(X(t), X(s)) \leq \delta(X,|t-s|)$ for any $t, s \in J$.

Here $\delta(X, \varepsilon)$ denotes the modulus of continuity of the set $X$.

In what follows we consider the differential equation

$$
x^{\prime}=f(t, x)
$$

with the initial condition

$$
x(0)=x_{0}
$$

We will assume that $f$ is defined and uniformly continuous on the set $J \times K\left(x_{0}, x\right)$ and take values in $E$. Furthermore, let $\omega(t, u)$ be a given Kamke comparison function belonging to $C$. We assume that $f$ satisfies the following comparison condition

$$
\mu(f(t, x)) \leq \omega(t, \mu(X))
$$

for every $X \in \dot{M}_{E}$ and for almost all $t \in J$.

Consider the function $\bar{\omega}: J \times R_{+} \rightarrow R_{+}$defined by the formula

$$
\bar{\omega}(t, u)=\sup \left[\mu(f(t, X)): \mu(X)=u, X \subset K\left(x_{0}, r\right)\right] .
$$

Observe that

$$
\bar{\omega}(t, u) \leq \omega(t, u)
$$

which follows directly from the definition of $\bar{\omega}(t, u)$ and (5). Next notice that monotonicity of $\mu$ and its continuity with respect to the Hausdorff distance (see Section 2) imply that $\bar{\omega}(t, u$ ) is nondecreasing with respect to $u$.

Further, let us take $u, u_{0} \in R_{+}$such that $0<u_{0}<u \leq r=\mu\left(K\left(x_{0}, r\right)\right)$. Assume that $t \in J$ is fixed. Take an 
arbitrarily small number $\varepsilon>0$ and let $K \subset K\left(x_{0}, r\right)$ be convex and such that $\mu(X)=u$ and $\bar{\omega}(t, u) \leq \mu(f(t, X))+\varepsilon$. Without loss of generality we may assume that $\theta \in X$. Consider the set $Y=\left(u_{0} / u\right) X$. Actually $Y$ is convex, $Y \subset X$ and $\mu(y)=u_{0}$. Moreover we have

$$
D(X, Y) \leq \frac{u-u_{0}}{u}\|X\|
$$

Hence we have

$\bar{\omega}(t, u)-\bar{\omega}\left(t, u_{0}\right) \leq \mu(f(t, X))-\mu(f(t, Y))+\varepsilon \leq D(f(t, X), f(t, Y))+\varepsilon$

which in view of Lemma 1 gives

$$
\bar{\omega}(t, u)-\bar{\omega}\left(t, u_{0}\right) \leq \delta\left(f\left(t, \frac{u-u_{0}}{u}\|X\|\right)\right)+\varepsilon,
$$

where we have denoted by $\delta(f(t, m))$ the modulus of continuity of the function $f(t, x)$ with respect to $x$. Finally, since $\varepsilon$ was chosen arbitrarily we have

$$
\bar{\omega}(t, u)-\bar{\omega}\left(t, u_{0}\right) \leq \delta\left(f\left(t, r\left(1-\frac{u_{0}}{u}\right)\right)\right) .
$$

In the same way we may carry over the proof of the following inequality

$$
\left|\bar{\omega}\left(t_{1}, u\right)-\bar{\omega}\left(t_{2}, u\right)\right| \leq \delta\left(\left|t_{1}-t_{2}\right|\right)
$$

where $\delta(m)$ denotes the modulus of continuity of the function $f(t, x)$ on the set $J \times K\left(x_{0}, r\right)$.

Now we may deduce the following theorem.

THEOREM 3. The function $\bar{\omega}(t, u)$ belongs to the class A.

Proof. Up to now we have shown that $\bar{\omega}$ is nondecreasing with respect to the second variable and continuous on the set $J \times(0,+\infty)$. In virtue of (6) and Theorem 2 it suffices to show that $\bar{\omega}(t, 0)=0$ for $t \epsilon J$ and $\bar{w}(t, u)$ is continuous for $u=0$. The first fact follows from the definition of $\bar{\omega}(t, u)$ and the property saying that the image of a compact set under a uniformly continuous function is a compact set. In order to prove the second assertion let us take $u>0, \varepsilon>0$ and a set $X$ such that $\mu(X)=u$ and $\bar{\omega}(t, u) \leq \mu(f(t, X))+\varepsilon$. In view of the definition 
of a measure $\mu$ we can find a finite set $\left\{x_{1}, x_{2}, \ldots, x_{n}\right\} \subset E$ such that $x \subset \bigcup_{i=1}^{n} K\left(x_{i}, u+\varepsilon\right)$. Thus, since $f$ is uniformly continuous we obtain

$$
\begin{aligned}
\bar{\omega}(t, u) & \leq \mu\left(f\left(t, \bigcup_{i=1}^{n} k\left(x_{i}, u+\varepsilon\right)\right)\right]+\varepsilon \leq \mu\left(\bigcup_{i=1}^{n} k\left(f\left(t, x_{i}\right), \delta(f(t, u+\varepsilon))\right)+\varepsilon\right) \\
& \leq \delta(f(t, u+\varepsilon))+\varepsilon
\end{aligned}
$$

which implies finally

$$
\bar{\omega}(t, u) \leq \delta(f(t, u)) .
$$

Obviously the desired continuity of the function $\bar{\omega}(t, u)$ for $u=0$ follows from the above inequality which completes the proof.

The theorem proved above shows that a lot of existence theorems for the Cauchy problem (3) and (4) in a Banach space are no more general than that given by Sadovskii [13], for instance. Moreover we are able to formulate an existence theorem being formally stronger than all those given up to now and involving an assumption of type [5].

THEOREM 4. Let $f: J \times K\left(x_{0}, r\right) \rightarrow E$ be a uniformly continuous junction, bounded by a constant $A$ and let the condition (5) be satisfied, where $\omega(t, u)$ is a Kamke comparison function from the class $C$. If $A T \leq r$ then there exists at least one solution of equation (3) satisfying condition (4) defined for $t \in J$.

The proof follows immediately from Theorem 3 and the earlier proved existence theorems.

Finally let us remark that Theorems 3 and 4 are also true in a more general situation when we consider measures of noncompactness defined in an axiomatic way. The detailed considerations will appear elsewhere.

\section{References}

[1] A. Ambrosetti, "Un teorema di esistenza per le equazioni differenziali negli spazi di Banach", Rend. Sem. Mat. Univ. Padova 39 (1967), 249-360. 
[2] Józef Banaś and Kazimierz Goebel, Measures of noncompactness in Banach spaces (Lecture Notes in Pure and Applied Mathematics, 60. Marcel Dekker, New York, 1980).

[3] Józef Banaś, Andrzej Hajnosz and Stanislaw Wedrychowicz, "Relations among various criteria of uniqueness for ordinary differential equations", Comment. Math. Univ. Carolin. 22 (1981), 59-70.

[4] E. Bompiani, "Un teorema di confronto ed un teorema di unicita per lequazione differenziale $y^{\prime}=f(x, y)$ ", Atti Accad. Naz.Lincei Rend. Cl. Sci. Fis. Mat. Natur. 1 (1925), 298-302.

[5] E. Coddington and N. Levinson, Theory of ordinary differential equations (McGraw-Hill, New York, 1955).

[6] Klaus Deimling, Ordinary differential equations in Banach spaces (Lecture Notes in Mathematics, 596. Springer-Verlag, Berlin, Heidelberg, New York, 1977).

[1] Klaus Deimling, "On existence and uniqueness for Cauchy's problem in infinite dimensional Banach spaces", Proc. Colloq. Math. Soc. Janos Bolyai Differential Equations 15 (1975), 131-142.

[8] Kazimierz Goebel and Witold Rzymowski, "An existence theorem for the equations $x^{\prime}=f(t, x)$ in Banach space", Buzz. Acad. Polon. Sci. Sér. Sci. Math. Astronom. Phys. 18 (1970), 367-370.

[9] K. Kuratowski, Topology I, new edition, revised and augmented (translated by J. Jaworowski. Academic Press, New York and London; Państwowe Wydawnictwo Naukowe, Warsaw; 1966).

[10] V. Lakshmikantham and S. Leela, Nonlinear differential equations in abstract spaces (Pergamon, New York, 1981).

[11] R.H. Martin, Nonlinear operators and differential equations in Banach spaces (John Wiley and Sons, New York and London, 1976).

[12] Czeslaw Olech, "Remarks concerning criteria for uniqueness of solutions of ordinary differential equations", Buzl. Acad. Polon. Sci. Sér. Sci. Math. Astronom. Phys. 8 (1960), 661-666.

[13] B.N. Sadovskii, "Limit compact and condensing mappings", Uspehi Mat. Nauk 27 (1972), 81-146. 
[14] Jacek Szarski, Differential inequalities (Monografie Matematyczne, 43. PWN - Polish Scientific Publishers, Warszawa, 1965).

[15] Stanislaw Szufla, "Measure of noncompactness and ordinary differential equations in Banach spaces", Bull. Acad. Polon. Sci. Sér. Sci. Math. Astronom. Phys. 19 (1971), 831-835.

[16] Wol fgang Walter, Differential and integral inequalities (SpringerVerlag, Berlin, Heidelberg, New York, 1970).

Institute of Mathematics and Physics, Technical University, 35-084 Rzeszów, Poznańska 2, Poland. 